\title{
Tratamientos pregerminativos para la ruptura de la dormición en semillas de tres poblaciones de Ramorinoa girolae, leñosa endémica de zonas áridas en Argentina
}

\author{
Pre-germinative treatments for seed dormancy breaking of three populations of \\ Ramorinoa girolae, an endemic woody species from arid zones in Argentina
}

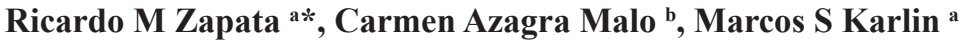 \\ *Autor de correspondencia: ${ }^{\text {a }}$ Universidad Nacional de Córdoba, Facultad de Ciencias Agropecuarias, Departamento Recursos \\ Naturales, Valparaíso S/N, Ciudad Universitaria, CC 509, CP 5000, Córdoba, Argentina, rzapata@agro.unc.edu.ar \\ ${ }^{\mathrm{b}}$ Universidad de Lleida, Departamento de Producción Vegetal y Ciencia Forestal, España.
}

\begin{abstract}
SUMMARY
For the conservation of species, especially those endemic or vulnerable, it is necessary to deepen the knowledge about their ecological dynamic and to find adequate techniques for multiplication. Ramorinoa girolae, "chica", is an endemic woody species of the xerophytic flora from western Argentine arid regions, with a restricted occupation area and fragmented communities. The lack of records makes relevant the conduction of research underpinning knowledge of the species, regeneration mechanisms and multiplication techniques. The objective of this work was to study the behavior of seeds of three populations under germination conditions and the response for different pre-germinative treatments. Germination trials were made in the laboratory with different pre-germinative techniques using the seeds of three populations under a completely aleatory design. Results confirm the presence of physical dormancy in seeds restricting the imbibing process for their rapid germination. The treatments, hot water scarification and mechanical scarification with sandpaper, increase significantly the germination percentages. The best treatment is mechanical scarification with sandpaper, reaching values of $92 \%$ of germination at five days from the beginning of the treatment. The uneven response of germination of the different populations confirms the different behavior of local populations and results interesting for ecosystem studies and future lines of investigation and management of this species. These technical aspects appear as relevant for the application of reforestation and environmental restoration programs.
\end{abstract}

Key words: dormancy, reforestation, pre-germinative treatments, endemic species, arid ecosystems.

\section{RESUMEN}

Para la conservación de las especies, especialmente endémicas o vulnerables, es necesario profundizar el conocimiento de su dinámica ecológica y encontrar técnicas adecuadas de multiplicación. Ramorinoa girolae, "chica”, es una especie leñosa endémica de la flora xerófila del oeste árido argentino, con restringida área de ocupación y distribución fragmentada. La falta de antecedentes hace relevante realizar investigaciones que fundamenten el conocimiento de la especie, los mecanismos de regeneración y las técnicas para su multiplicación. El objetivo de este trabajo fue estudiar el comportamiento de semillas de tres poblaciones en condiciones de germinación y la respuesta a diferentes tratamientos pregerminativos. Se realizaron ensayos de germinación en laboratorio con distintas técnicas pregerminativas utilizando semillas de tres poblaciones bajo un diseño completamente aleatorizado. Los resultados confirman la presencia de dormición física en las semillas que restringen el proceso de imbibición para su rápida germinación. Los tratamientos ensayados, escarificación con agua caliente y mecánica con lija, incrementan significativamente los porcentajes de germinación. El tratamiento que produce mejores resultados es la escarificación mecánica con lija, alcanzando valores del $92 \%$ de germinación a los cinco días de comenzado el tratamiento. La desigual respuesta de germinación de distintas poblaciones confirma el comportamiento diferente de poblaciones locales que resulta de interés en los estudios ecosistémicos y futuras líneas de investigación y gestión de esta especie. Estos conocimientos son relevantes en la propagación de la especie y la aplicación de programas reforestación y restauración ambiental.

Palabras clave: dormición, reforestación, tratamientos pregerminativos, especie endémica, ecosistemas áridos.

\section{INTRODUCCIÓN}

El germoplasma de las poblaciones vegetales que se desarrollan en condiciones de aridez se encuentra frecuentemente amenazado por la conjunción de condiciones am- bientales, tales como el marcado déficit hídrico, altas temperaturas, suelos descubiertos (Solh y van Ginkel 2014) y factores relacionados con la gestión, como la falta de políticas ambientales, tala indiscriminada de las especies leñosas y el sobrepastoreo; dificultando las posibilidades 
de estas poblaciones naturales para regenerarse y acrecentando el riesgo de su extinción (Javed et al. 2013).

Los estudios sobre la recolección y conservación de germoplasma de poblaciones vegetales naturales, su multiplicación, manejo y la valoración de la variabilidad genética, constituyen la base para su posterior uso, domesticación y manejo, evitando así procesos de erosión genética (Jaramillo y Baena 2000). Para la conservación de una especie es necesaria su identificación, caracterización y profundizar el conocimiento de su dinámica ecológica, con el fin de encontrar las técnicas más adecuadas de multiplicación para obtener renuevos en vivero y poder realizar a posterio$r i$ ensayos de reforestación (Villagra et al. 2004, Villarreal Garza et al. 2013), lo cual resulta de especial relevancia en aquellas especies endémicas, vulnerables o amenazadas.

El endemismo surge como consecuencia de la especialización provocada ante la presencia de barreras naturales que impiden el intercambio genético, apareciendo especies restringidas a esas zonas geográficas (Godoy-Bürki et al. 2014). Estas especies son vulnerables, pues sus poblaciones suelen tener reducido número de individuos y acervos génicos así como limitadas capacidades de adaptación a nichos ambientales diferentes (Brooks et al. 2002). Es por ello que las especies endémicas requieren especial atención en cuanto a su conservación y la necesidad de revalorizar la potencialidad de sus funciones ecológicas y productivas (Casas y Parra 2007).

Ramorinoa girolae Speg., "chica” (Fabaceae, tribu Dalbergiae), es una especie leñosa endémica característica de la flora xerófila del oeste árido argentino (Cangiano et al. 1998). Habita en el cuadrante delimitado por los paralelos $29^{\circ} 30^{\prime}$ y $32^{\circ} 30^{\prime} \mathrm{S}$ y los meridianos $67^{\circ} 00^{\prime}$ y $68^{\circ}$ $05^{\prime} \mathrm{O}$, entre los 700 y los $2.400 \mathrm{~m}$ s.n.m. (Zapata et al. 2009). La especie integra un género monotípico de la tribu Dalbergiae (Subils 1983), dentro de la cual se incluyen especies leñosas económicamente importantes por sus maderas duras (Dalbergia spp. y Pterocarpus spp.), por sus cualidades forrajeras (Stylosanthes spp.) y propiedades industriales (Arachis spp.) (Lavin et al. 2001). La madera de esta especie es usada como combustible por su alto poder calorífico y para la confección de artesanías y utensilios rurales por su gran dureza y resistencia (Demaio et al. 2015), siendo sus semillas comestibles y empleadas ancestralmente como alimento por las poblaciones humanas locales (Gómez Sosa 1994). Por ser fuente de componentes antioxidantes y poseer alto contenido de aceites oleicos y potasio, estas semillas pueden constituir una opción valiosa para la alimentación humana (Luna et al. 2013).

Ramorinoa girolae es una de las pocas especies leñosas arbóreas que se desarrolla en la Región Fitogeográfica del Monte (Demaio et al. 2015) la cual posee características climáticas áridas. Su endemismo, su función en el ecosistema y la potencialidad de uso alimenticio por parte de comunidades rurales, hacen necesario el inicio de investigaciones que revelen su comportamiento ecológico para su gestión y conservación. Su restringida área de ocu- pación así como el agrupamiento de individuos que conforman comunidades vegetales fragmentadas y distribuidas a manera de islas (Hadad et al. 2014), pueden definir poblaciones locales con características y comportamientos ecofisiológicos diferentes (Morlans 2004). La falta de antecedentes en la materia hace relevante la publicación de investigaciones que fundamenten el conocimiento de la especie para su uso sustentable. Así, se consideran prioritarias las indagaciones sobre los mecanismos de regeneración, germinación de semillas y la aplicación de técnicas para su multiplicación.

Debido a la presencia de latencia, no todas las semillas colocadas bajo condiciones favorables de germinación pueden generar plántulas; según Doria (2010), hay varias causas que determinan la dormición en las semillas, entre ellas la presencia de embriones rudimentarios o fisiológicamente inmaduros, la resistencia mecánica de las cubiertas seminales o la impermeabilidad de las mismas, la presencia de inhibidores, etc. Las especies leguminosas se caracterizan por presentar testa dura, condición que inhibe temporariamente la germinación. Para mejorar este proceso y acelerar la obtención de plántulas, existen diversos tratamientos pregerminativos, siendo los métodos más comúnmente empleados las escarificaciones mecánica, química y física, entre otras (Kimura e Islam 2012). Estas técnicas han sido empleadas en varias especies leñosas de los géneros Prosopis y Acacia, que habitan también las regiones áridas de argentina (Villagra et al. 2004).

La mayoría de las semillas de leguminosas silvestres de la misma tribu que la chica, tales como los géneros Dalbergia (Parrotta 1989) y Stylosanthes (Castillo y Guenni 2001), poseen algún grado de reposo, letargo o dormición, que normalmente se interrumpe cuando se presentan las condiciones adecuadas para la germinación (Sánchez-Paz y Ramírez-Villalobos 2006). Para el caso de $R$. girolae, son escasos los antecedentes bibliográficos en general de la especie y de experiencias registradas sobre técnicas para su multiplicación.

La hipótesis del trabajo es que las semillas de $R$. girolae poseen dormición, mecanismo que debe ser superado a través de tratamientos pregerminativos para lograr una rápida germinación y un stand de plantas homogéneo en la etapa de vivero. Al conformar poblaciones locales fragmentadas, sus comportamientos fisiológicos incluida la germinación son diferentes.

El objetivo de este trabajo es estudiar el comportamiento de semillas de tres poblaciones de $R$. girolae puestas en condiciones de germinación y la respuesta de las mismas a diferentes tratamientos pregerminativos, para avanzar en el conocimiento de la dinámica ecológica de la especie y contribuir a su uso sustentable y conservación.

\section{MÉTODOS}

Ramorinoa girolae es una especie de porte arbustivo o arbóreo de hasta 10 metros de altura, de copa globosa, 
áfila, con tallos fotosintetizantes cerosos que le otorgan las características xerófilas. Las flores, reunidas en racimos axilares de color amarillo, dan origen a un fruto en forma de legumbre leñosa extremadamente dura y resistente (Demaio et al. 2015). Cada inflorescencia origina entre 1 y 3 legumbres de maduración estivo-otoñal, secas, leñosas y fibrosas e indehiscentes, pudiendo permanecer las semillas en el interior del fruto por largo tiempo (Gómez Sosa 1994)(figura 1).

Su hábitat está caracterizado por precipitaciones de ocurrencia estival (entre noviembre y marzo) que varían entre 100 y $350 \mathrm{~mm}$, de carácter torrencial y con una marcada variabilidad temporal y espacial (Abraham et al. 2009). La relación entre las precipitaciones y la evapotranspiración potencial oscila entre 0,05 y 0,5 . El área presenta, además, una gran amplitud térmica anual, siendo la temperatura media del mes más cálido (enero) de 25 ${ }^{\circ} \mathrm{C}$ y la del mes más frío (julio) de $11^{\circ} \mathrm{C}$, con variaciones locales en función de la altitud (Labraga y Villalba 2009).

Dentro del área de distribución de la especie (figura 2), se seleccionaron tres poblaciones locales (Morlans 2004), en diferentes posiciones geográficas distanciadas a más de $40 \mathrm{~km}$ entre ellas y separadas por barreras orográficas. En el mes de abril, estando los frutos maduros, se colectaron las legumbres de la copa de un conjunto de árboles en las tres poblaciones seleccionadas que fueron:

1. Población $\mathrm{N}^{\circ} 1$ : Talampaya. $29^{\circ} 40^{\prime} \mathrm{S} ; 67^{\circ} 44^{\prime} \mathrm{O}$

2. Población $\mathrm{N}^{\circ}$ 2: Ischigualasto. $30^{\circ} 12^{\prime} \mathrm{S} ; 67^{\circ} 49^{\prime} \mathrm{O}$

3. Población $\mathrm{N}^{\circ} 3$ : Vilgo. $29^{\circ} 56^{\prime} \mathrm{S} ; 7^{\circ} 23^{\prime} \mathrm{O}$

Los frutos fueron abiertos individualmente realizando un corte lateral mediante una sierra de metal, extrayéndose posteriormente las semillas contenidas en su interior. Se realizó una selección de las semillas, descartando las daña- das, perforadas o defectuosas. Del conjunto de semillas de cada población se separaron para cada tratamiento, cuatro grupos (repeticiones) de 25 semillas cada uno, según recomendaciones del Manual para Manejo de Semillas (Rao et al. 2007). Los tratamientos realizados fueron:

A) Condición testigo (T0): Las semillas no recibieron ningún tratamiento pregerminativo previo a la colocación en la cámara de germinación.

B) Tratamientos pregerminativos:

T1: Inmersión en agua caliente a temperatura inicial de $80{ }^{\circ} \mathrm{C}$ durante 24 horas. Cada lote de 100 semillas, de cada una de las tres poblaciones, se colocó en un recipiente de vidrio donde se vertió agua caliente a temperatura inicial de $80{ }^{\circ} \mathrm{C}$, dejando que la temperatura alcanzara progresivamente la temperatura ambiente $\left(20^{\circ} \mathrm{C}+/-1^{\circ} \mathrm{C}\right)$.

$\mathrm{T} 2$ : Inmersión en agua a temperatura ambiente $\left(20^{\circ} \mathrm{C}\right)$ durante 24 horas. Para el tratamiento con agua a temperatura ambiente se siguió el mismo procedimiento que en el apartado anterior, quedando las semillas sumergidas durante 24 horas en agua a $20^{\circ} \mathrm{C}+/-1{ }^{\circ} \mathrm{C}$.

T3: Escarificación mecánica. Abrasión de la semilla con lija. Las semillas fueron sometidas en forma individual a la acción abrasiva de papel de lija de grano mediano $\mathrm{N}^{\circ} 80$ (Normas ANSI y FEPA "P"), en forma manual, presionando y arrastrando la semilla sobre la lija por la parte opuesta de la región micropilar.

En todos los casos las semillas fueron tratadas posteriormente con una solución de hipoclorito de sodio al $5 \%$
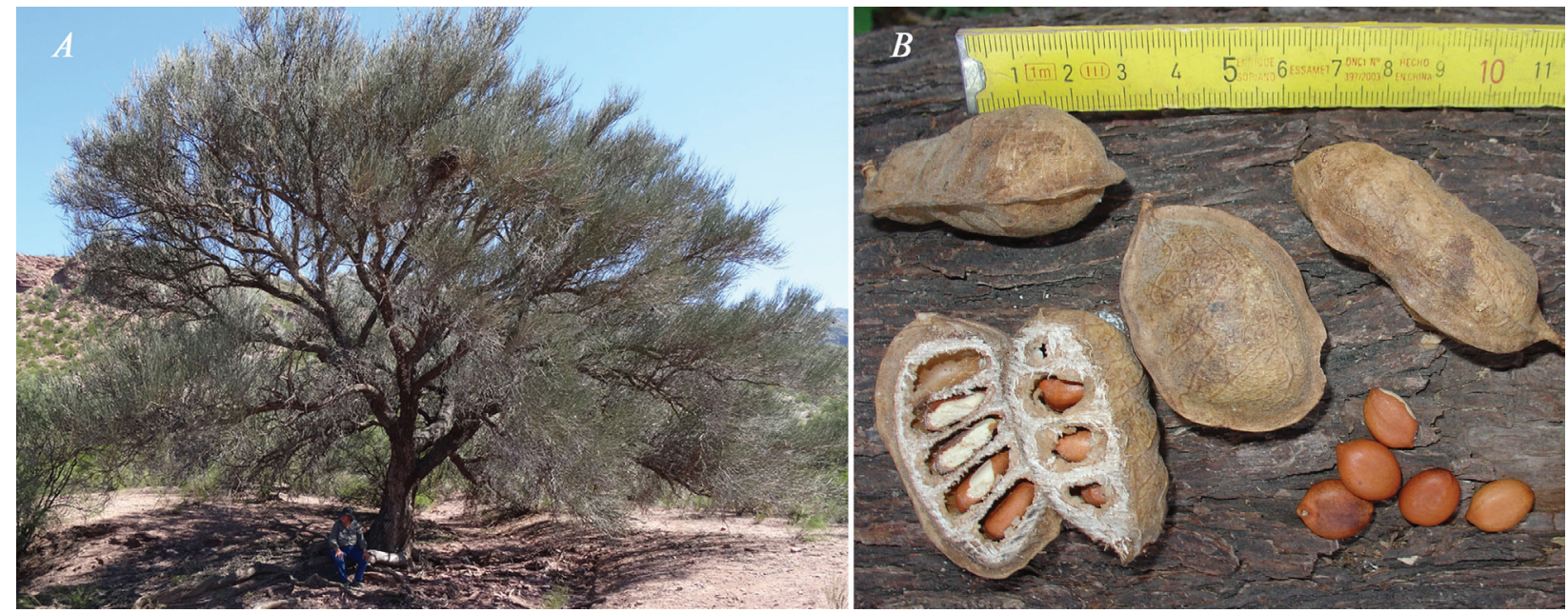

Figura 1. A) Ejemplar adulto de "chica"; B) Detalle de frutos y semillas.
A) Adult specimen of the "chica".
B) Detail of fruits and seeds. 


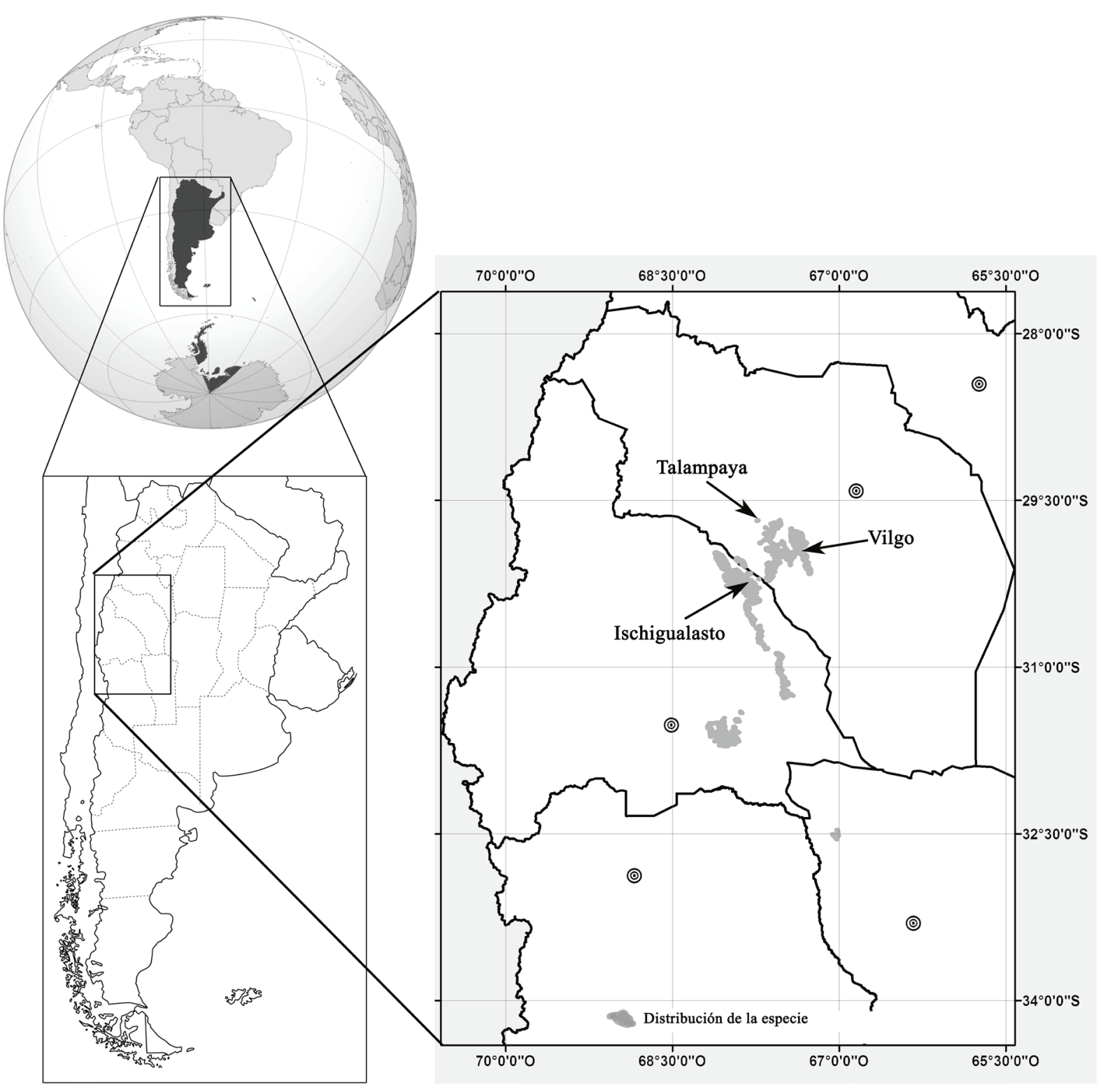

Figura 2. Área de distribución de Ramorinoa girolae en Argentina y poblaciones seleccionadas.

Distribution area of Ramorinoa girolae in Argentina and selected populations.

a los efectos de prevenir la aparición de enfermedades fúngicas. A continuación las semillas de cada repetición se colocaron sobre papel de filtro doble hoja previamente humedecido sobre una bandeja plástica y dentro de bolsas de nylon para mantener la humedad. Posteriormente las bandejas fueron colocadas en cámara de germinación a temperatura alterna de $20-30{ }^{\circ} \mathrm{C}$ y ciclo de luz-oscuridad de 8 y 16 h, respectivamente (ISTA 1999), en el Laboratorio de Semillas de la Facultad de Ciencias Agropecuarias, Universidad Nacional de Córdoba.

Para la evaluación de las semillas en la germinación en laboratorio se aplicaron los protocolos indicados en las Normas ISTA $(1999,2003)$. Considerando las mismas, se adoptó para el presente trabajo el concepto de germinación de la semilla como el comienzo de la aparición de la raíz tras romper la cubierta seminal y a lo largo de 30 días se efectuó el recuento de semillas germinadas, duras, hinchadas por imbibición y muertas.

La variable respuesta en todos los tratamientos fue la germinación de la semilla evaluada por el registro de la presencia de la radícula por fuera de la cubierta seminal, contabilizándose la cantidad de semillas germinadas sobre el total.

Los ensayos de germinación en laboratorio se llevaron a cabo bajo un diseño completamente aleatorizado. Los datos expresados en porcentajes de germinación fueron transformados aplicando la raíz cuadrada del arco-seno a fin de normalizar su distribución y sobre estos valores 
se realizó la evaluación mediante análisis de la varianza (ANDEVA), evaluando la respuesta entre tratamientos e interacción entre estos y la población. Las diferencias entre las medias se sometieron a una prueba de comparación a posteriori utilizando la prueba de Tukey, considerando un nivel de significación para una $P<0,05$. Para la interacción población y tratamiento, se aplicó un test DGC $(P<0,05)$ (Di Rienzo et al. 2002) a fin de exponer diferencias significativas entre cada interacción. Para el análisis estadístico se utilizó el programa InfoStat (Di Rienzo et al. 2012).

La caracterización del proceso germinativo se evaluó gráficamente mediante curvas de germinación acumulada, en las cuales se visualizaron los índices que se han usado para estudiar numéricamente el fenómeno.

\section{RESULTADOS}

Condición testigo. En las semillas de la condición testigo se observó que durante los primeros cinco días se producía el hinchamiento solo de menos del $5 \%$ de las semillas, evidenciado por un aumento notorio de su tamaño original, correspondiendo este fenómeno a la etapa de imbibición. Para aquellas semillas que mostraron cambio de tamaño por efecto de este fenómeno, el inicio de la manifestación comenzó a partir del día 2 de colocadas en el papel filtro humedecido, permaneciendo en este estado por 24 a $48 \mathrm{~h}$, a partir de las cuales se produjo la emergencia del ápice radical por el extremo de las semillas (figura 3 ).

El proceso de emergencia de la radícula (figura 4) se manifestó durante los 30 días de observación, ocurriendo solo en una cantidad muy baja de las semillas en los primeros 10 días del proceso (menos del $20 \%$ ). Entre los 15 y 20 días se registró el mayor porcentaje de germinación, decayendo a partir de este periodo el número de nuevas semillas germinadas, hasta llegar al día 30 donde finalizó el ensayo. Al finalizar el período de observación, las semillas no germinadas permanecían en su estado original, consecuentemente no se produjo el ingreso de agua a su interior y no se inició el proceso de imbibición.

Las tres poblaciones respondieron de manera similar en la cantidad de semillas germinadas hasta transcurridos 15 días del inicio de la experiencia. A partir de este lapso, la población de Vilgo logró un salto diferencial con respecto a las otras poblaciones con un porcentaje de germinación mayor al $50 \%$. Al final del periodo de evaluación se observaron diferencias significativas $(P=0,0029)$ en el poder germinativo entre las poblaciones evaluadas. La prueba de Tukey diferenció la población de Vilgo, con mayor porcentaje de germinación, respecto a las de Talampaya e Ischigualasto.

Tratamientos pregerminativos. Los resultados muestran diferencias significativas $(P<0,001)$ entre los tratamientos aplicados, sobre el conjunto total de semillas de las tres poblaciones analizadas (figura 5).

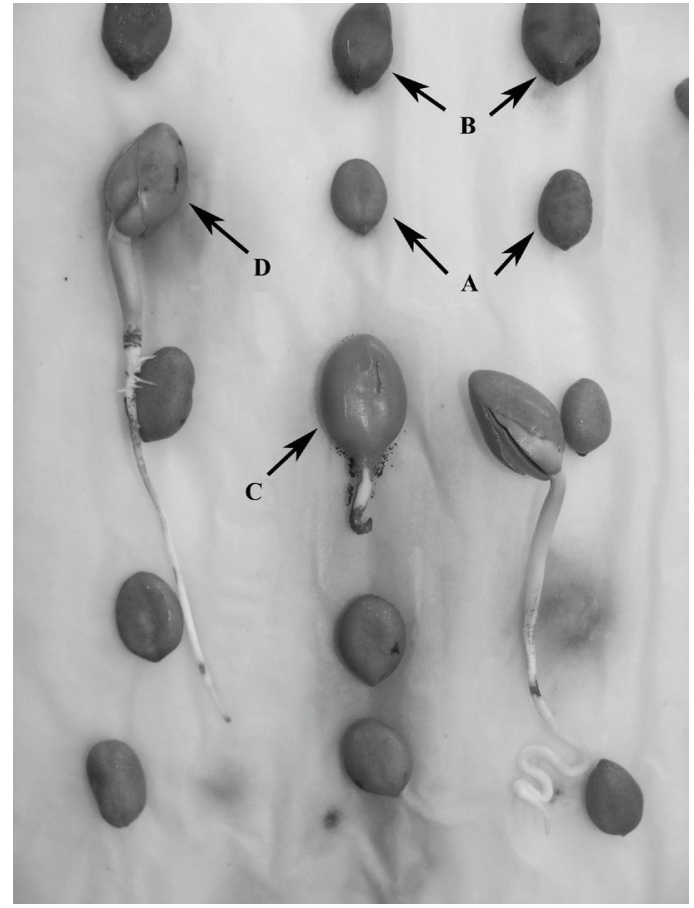

Figura 3. Desigualdad en el proceso de germinación en la condición testigo (T0) a los 15 días de comenzado el tratamiento. A) Semillas normales. B) Semillas hinchadas por imbibición. C) Semillas con emergencia de radícula incipiente. D) Semillas con raíz desarrollada y aparición de cotiledones.

Non-uniformity in the germination process in the control condition (T0) 15 days after the beginning of the treatment. a) Normal seeds. b) Swollen seeds by imbibition. c) Seeds with incipient radicle emergency. d) Seeds with developed root and cotyledon appearance.

La escarificación mecánica ofrece los mayores porcentajes de germinación, obteniéndose valores promedio del $92 \%$. Más del $70 \%$ de las semillas germinaron al cabo del quinto día en comparación a los otros tratamientos y al testigo, todos ellos con menos del $20 \%$ a la misma fecha.

El tratamiento con agua caliente mejora significativamente el porcentaje de germinación respecto al testigo, alcanzando el $70 \%$, aunque el período de emergencia de las radículas es más lento respecto al escarificado mecánico con lija; 20 días más para el logro de la misma cantidad de semillas germinadas.

Las semillas remojadas con agua a temperatura ambiente durante 24 horas alcanzan a los 30 días un $55 \%$ de germinación, no mostrando diferencias significativas con el testigo, pero sí con las remojadas en agua caliente a $80^{\circ} \mathrm{C}$ y escarificadas con lija.

En el tratamiento de escarificación mecánica, a partir de los 10 días de ensayo no se observó ninguna nueva semilla germinada. En los otros tratamientos y el testigo, a partir de los 20 días la cantidad de nuevas semillas germinadas disminuye notablemente, produciendo la caída y estabilización de la curva que representa la germinación acumulada. 


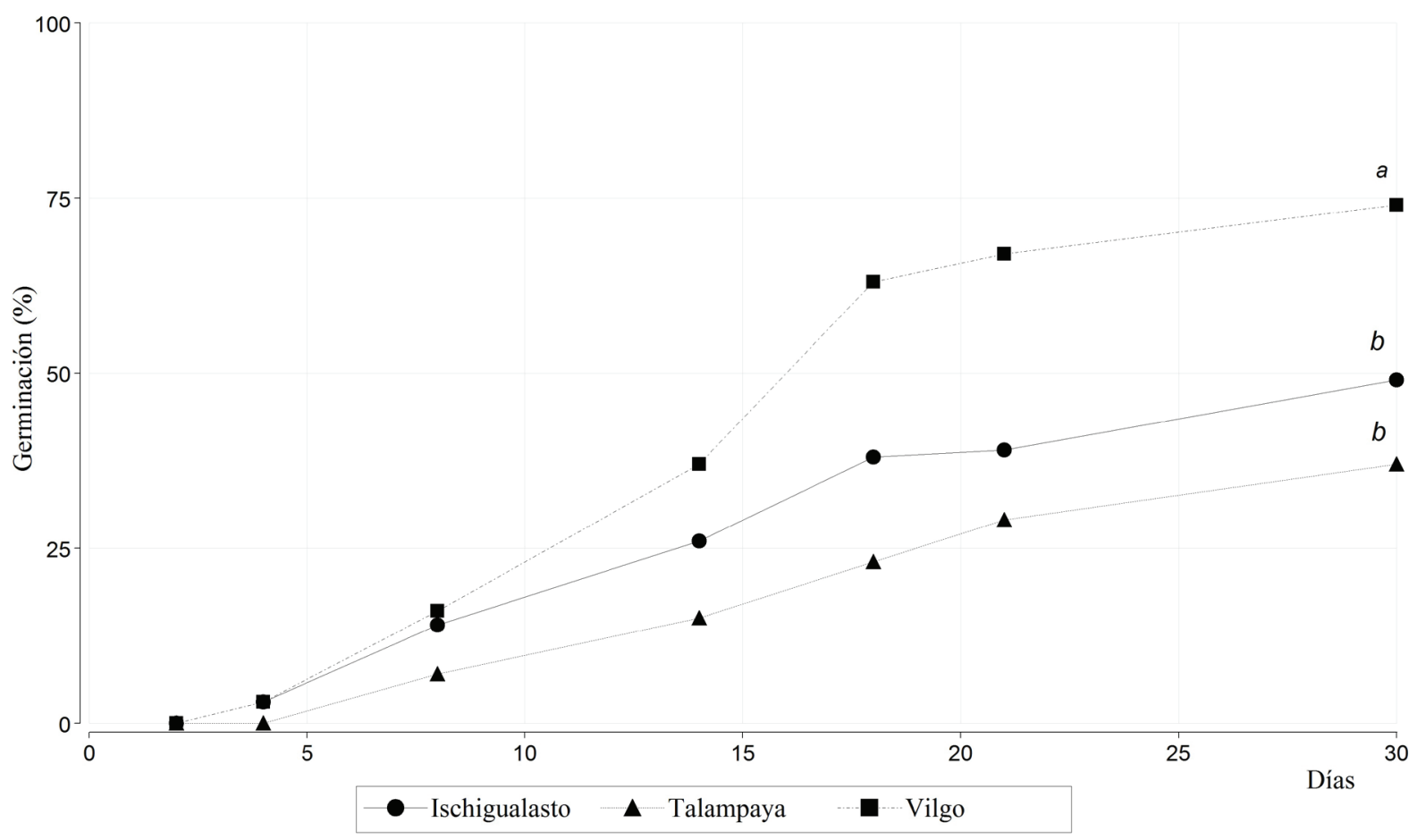

Figura 4. Porcentaje promedio de germinación en la condición testigo según poblaciones. Letras distintas indican diferencias significativas (Tukey $P<0,05$ ).

Germination percentage in chamber by population. Different letters indicate significant differences (Tukey $P<0.05$ ).

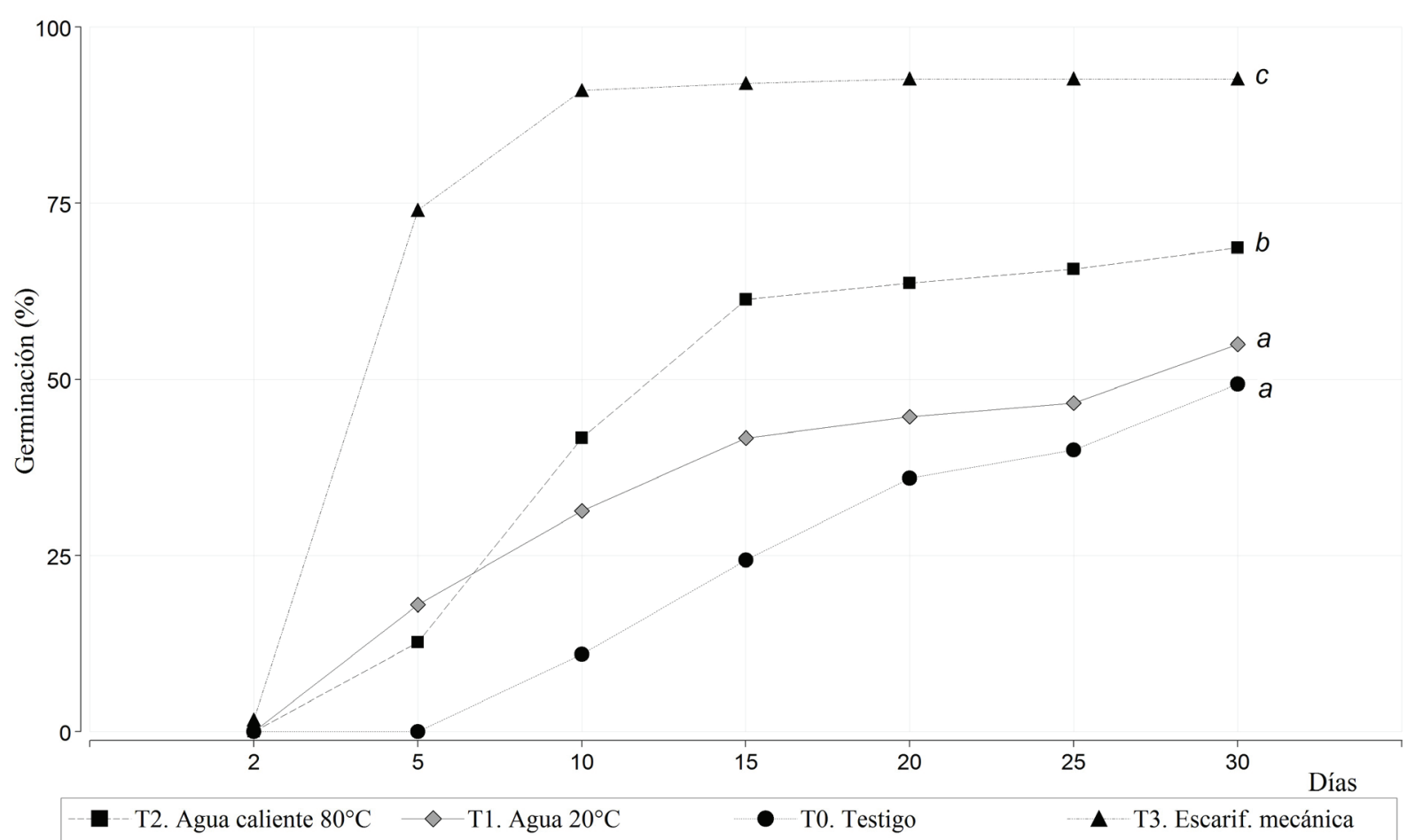

Figura 5. Porcentaje de germinación según los distintos tratamientos pregerminativos. Letras distintas indican diferencias significativas (Tukey $P<0,05$ ).

Germination percentage by pre-germinative treatment. Different letters indicate significant differences (Tukey $P<0.05$ ). 
Las poblaciones de Ischigualasto y Vilgo presentaron mejor respuesta de germinación en los tratamientos pregerminativos, a diferencia de la población de Talampaya que, tanto para la condición testigo como los tratamientos de inmersión en agua, mostró los valores más bajos de germinación. La escarificación promueve altos y similares porcentajes de germinación para las tres poblaciones.

$\mathrm{Si}$ se analizan las interacciones, puede apreciarse en la figura 6 que se presentan diferencias significativas $(P<0,001)$ entre las poblaciones orígenes de la semilla y los tratamientos.

\section{DISCUSIÓN}

Los resultados de germinación confirman la presencia del estado de dormición física en las semillas, impuestas por la impermeabilidad de los tegumentos que restringen el proceso de imbibición, tal como ocurre en muchas especies de la familia Fabaceae, demostrado por varios autores (Muñoz et al. 2009, Villarreal Garza et al. 2013). Consecuentemente, a los efectos de favorecer el inicio del proceso germinativo en $R$. girolae, es necesario el empleo de técnicas que rompan esta barrera.

De los tratamientos seleccionados, la acción abrasiva de la lija, como escarificación mecánica, acelera la pene- tración del agua al interior de la semilla, desencadenando el proceso de imbibición y una rápida germinación. El ablandamiento de las cubiertas con agua caliente, utilizado como tratamiento exitoso con otras leguminosas (Sánchez-Paz y Ramírez-Villalobos 2006, Muñoz et al. 2009), produce menores porcentajes de germinación que el tratamiento anterior, aunque mejora la velocidad del proceso y el porcentaje final respecto al testigo.

Se observa disparidad en la germinación de la población de Talampaya respecto a las otras dos poblaciones, tanto en la condición testigo como en los tratamientos de inmersión en agua, equiparándose los resultados en el tratamiento de escarificación mecánica. Esta situación sugiere la presencia de una cubierta seminal de mayor resistencia a la penetración del agua en los individuos de esta población, tal como lo encontraron Castillo y Guenni (2001) en Stylosanthes hamata (L.) Taub., quienes afirman que este tipo de latencia puede variar de acuerdo al origen del genotipo y representa una propiedad adaptativa que promueve la sobrevivencia de las plantas bajo condiciones adversas. Dicha resistencia se rompe igualmente en las semillas de todas las poblaciones mediante la escarificación mecánica.

Las semillas remojadas a temperatura ambiente durante 24 horas y el lote testigo muestran una imbibición diferencial y no uniforme de las semillas, espaciando en el

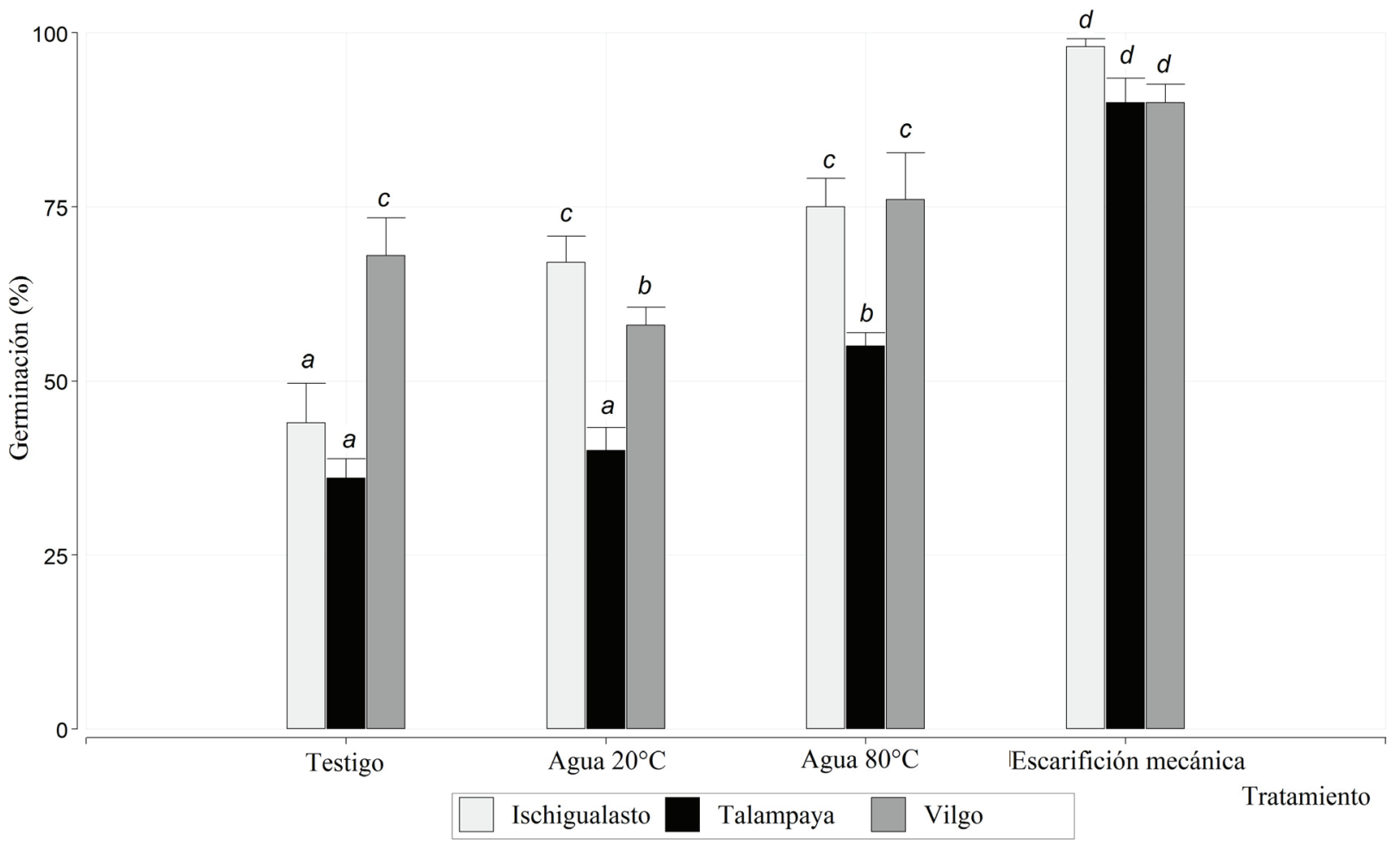

Figura 6. Porcentaje de germinación final según tratamiento y población a los 30 días. Letras diferentes indican diferencias significativas $(P<0,001)$ de la interacción entre población y tratamiento.

Final germination percentage by treatment and population at 30 days. Different letters indicate significant differences $(P<0.001)$ between population and treatment interaction. 
tiempo el inicio del proceso de germinación y limitando la cantidad de semillas germinadas en el período evaluado. Esta latencia ofrece la posibilidad de regular naturalmente la germinación de las semillas de esta especie como estrategia de oportunidad de instalación de nuevos individuos a campo (Castillo y Guenni 2001).

Si bien no existen diferencias significativas entre el tratamiento con inmersión en agua a temperatura ambiente y el testigo, puede apreciarse mayor número de semillas germinadas y una aceleración en los períodos de germinación.

Los mayores porcentajes de germinación se observaron con la escarificación mecánica con lija, asociado a un tiempo de germinación menor, siendo este tratamiento el más eficiente para la obtención de plantines. Esta técnica permite reducir y uniformizar el tiempo de germinación, lograr un stand de plantas óptimo frente a la cantidad de semilla empleada y reducir los costos que implica la realización de plantines en vivero, asegurando la obtención del número de plantas necesario en el menor tiempo posible y con la mayor uniformidad.

\section{CONCLUSIONES}

Los resultados obtenidos muestran la existencia de un estado de dormición en la semilla de Ramorinoa girolae que debe ser interrumpido para su rápida germinación.

Los tratamientos pregerminativos ensayados, escarificación con agua caliente y mecánica con lija, incrementan significativamente los porcentajes de germinación, indicando la existencia de barreras impermeables en la semilla como mecanismos de latencia natural.

El tratamiento que produce los mejores resultados en el proceso de germinación de la semilla de $R$. girolae, número de semillas germinadas y velocidad de ocurrencia, es la escarificación mecánica con lija, alcanzando valores del $92 \%$ de germinación a los cinco días de comenzado el tratamiento.

La desigual respuesta de germinación en semillas de distintas poblaciones, confirma el comportamiento diferente de poblaciones locales que puede resultar de interés en los estudios ecosistémicos y en otras líneas de investigación y gestión de esta especie.

Los resultados de este trabajo permiten mejorar el conocimiento de esta especie en general, necesarios para la aplicación de técnicas de manejo en programas de conservación, reforestación o restauración ambiental.

\section{REFERENCIAS}

Abraham E, HF del Valle, F Roig, L Torres, JO Ares, F Coronato, R Godagnone. 2009. Overview of the geography of the Monte Desert biome (Argentina). Journal of Arid Environments 73(2): 144-153.

Brooks TM, RA Mittermeier, CG Mittermeier, GAB Da Fonseca, AB Rylands, WR Konstant, P Flick, J Pilgrim, S Oldfield, G Magin, C Hilton-Taylor. 2002. Habitat loss and extinction in the hotspots of biodiversity. Conservation Biology 16: 909-923.
Cangiano MA, L Zanín, H Losinno. 1998. El cariotipo de Ramorinoa girolae (Fabaceae). Kurtziana 26: 173-177.

Casas A, F Parra. 2007. Agrodiversidad, parientes silvestres y cultura. LEISA 23(2): 5-8.

Castillo R, O Guenni. 2001. Latencia en semillas de Stylosanthe hamata (Leguminosae) y su relación con la morfología de la cubierta seminal. Revista de Biología Tropical 49(1): 287-299.

Demaio P, U Karlin, M Medina. 2015. Árboles nativos de Argentina. Tomo 1: Centro y Cuyo. Córdoba, Argentina. Ecoval Ediciones. $188 \mathrm{p}$.

Di Rienzo JA, AW Guzmán, F Casanoves. 2002. A multiplecomparisons method based on the distribution of the root node distance of a binary tree. Journal of Agricultural, Biological, and Environmental Statistics 7(2): 129-142.

Di Rienzo JA, F Casanoves, MG Balzarini, L Gonzalez, M Tablada, CW Robledo. 2012. InfoStat versión 2012. Grupo InfoStat, FCA, Universidad Nacional de Córdoba. Consultado 9 jun. 2014. Disponible en http://www.infostat.com.ar

Doria J. 2010. Generalidades sobre las semillas: su producción, conservación y almacenamiento. Cultivos Tropicales 31(1): 74-85.

Godoy-Bürki AC, P Ortega-Baes, JM Sajama, L Aagesen. 2014. Conservation priorities in the Southern Central Andes: mismatch between endemism and diversity hotspots in the regional flora. Biodiversity and Conservation 23(1): 81-107.

Gómez Sosa E. 1994. Ramorinoa spegazzini. In Kiesling R ed. Flora de San Juan. Vol.1. Leguminoseae. Buenos Aires, Argentina. Vázquez Mazzini. p. 331-332.

Hadad M, M Almiron, J Scaglia. 2014. Estructura de un bosque de Ramorinoa girolae (Fabaceae), en la Sierra de Pie de Palo, San Juan (Argentina). Boletín de la Sociedad Argentina de Botánica 49(2): 283-292.

ISTA (International Seed Testing Association, CH). 1999. International Rules for Seed Testing. Seed Science and Technology 27 (suplemento): 1-333.

ISTA (International Seed Testing Association, CH). 2003. International Rules for Seed Testing. Edición 2003. Basserdorf, Switzerland. ISTA.

Jaramillo S, M Baena. 2000. Material de apoyo a la capacitación en conservación ex situ de recursos fitogenéticos. Cali, Colombia. Instituto Internacional de Recursos Fitogenéticos (IPGRI). $210 \mathrm{p}$.

Javed SB, M Anis, PR Khan, IM Aref. 2013. In vitro regeneration and multiplication for mass propagation of Acacia ehrenbergiana Hayne: a potential reclaiment of denude arid lands. Agroforestry Systems 87(3): 621-629.

Kimura E, MA Islam. 2012. Seed scarification methods and their use in forage legumes. Research Journal of Seed Science 5(2): 38-50.

Labraga JC, R Villalba. 2009. Climate in the Monte Desert: past trends, present conditions, and future projections. Journal of Arid Environments 73(2): 154-163.

Lavin M, RT Pennington, BB Klitgaard, JI Sprent, HC de Lima, PE Gasson. 2001. The dalbergioid legumes (Fabaceae): delimitation of a pantropical monophyletic clade. American Journal of Botany 88(3): 503-533.

Luna, LC, NB Pigni, L Torras-Claveria, MV Monferran, D Maestri, DA Wunderlin, GE Feresin, J Bastida, A Tapia. 2013. Ramorinoa girolae Speg (Fabaceae) seeds, an argentinean traditional indigenous food: Nutrient composition and anti- 
oxidant activity. Journal of Food Composition and Analysis 31: $120-128$.

Morlans MC. 2004. Introducción a la ecología de poblaciones. Catamarca, Argentina. Editorial Científica Universitaria. Universidad Nacional de Catamarca. 16 p.

Muñoz BC, JA Sánchez, LA Montejo, Y González, J Reino. 2009. Valoración germinativa de 20 accesiones de leguminosas almacenadas en condiciones desfavorables. Pastos y Forrajes 32(3): 1-15.

Parrotta JA. 1989. Dalbergia sissoo Roxb. Sissoo, Indian rosewood: Leguminosae (papilionoideae), Legume Family. Río Piedras, San Juan. Puerto Rico. U.S. Forest Service, Southern Forest Experiment Station, Institute of Tropical Forestry. 5 p.

Rao NK, J Hanson, ME Dulloo, K Ghosh, D Nowell, M Larinde. 2007. Manual para el manejo de semillas en Bancos de Germoplasma. Roma, Italia. Bioversity International. 165 p.

Sánchez-Paz Y, M Ramírez-Villalobos. 2006. Tratamientos pregerminativos en semillas de Leucaena leucocephala (Lam.) de Wit. y Prosopis juliflora (Sw.) DC. Revista de la Facultad de Agronomía de la Universidad de Zulia 23(3): 257-272.
Solh M, M van Ginkel. 2014. Drought preparedness and drought mitigation in the developing world's drylands. Weather and Climate Extremes 3: 62-66.

Subils R. 1983. Recuentos cromosómicos en Ramorinoa girolae (Fabaceae). Kurtziana 16: 166-167.

Villagra PE, MA Cony, NG Mantován, BE Rossi, MM González Loyarte, R Villalba, L Marone. 2004. Ecología y manejo de los algarrobales de la Provincia Fitogeográfica del Monte. In Arturi MF, JL Frangi, JF Goya eds. Ecología y manejo de bosques nativos de Argentina. La Plata, Argentina. Editorial Universidad Nacional de La Plata. Presentación multimedia en CD.

Villarreal Garza JA, A Rocha Estrada, ML Cárdenas-Ávila, S Moreno Limón, M González Álvarez, V Vargas López. 2013. Caracterización morfométrica, viabilidad y germinación de semillas de Mezquite y Huizache en el noreste de México. Phyton 82(2): 169-174

Zapata RM, C Azagra Malo, AM Lattandi. 2009. Distribución de poblaciones naturales de "Chica" (Ramorinoa girolae Speg., Fabaceae) Especie Endémica de Argentina. Boletín de la Sociedad Argentina de Botánica 44 (Supl.): 105.

Recibido: 16.09 .16

Aceptado. 18.01.17 
